\title{
Epigenomic mapping and effect sizes of noncoding variants associated with psychotropic drug response
}

\begin{abstract}
Aim: To provide insight into potential regulatory mechanisms of gene expression underlying addiction, analgesia, psychotropic drug response and adverse drug events, genome-wide association studies searching for variants associated with these phenotypes has been undertaken with limited success. We undertook analysis of these results with the aim of applying epigenetic knowledge to aid variant discovery and interpretation. Methods: We applied conditional imputation to results from 26 genome-wide association studies and three candidate gene-association studies. The analysis workflow included data from chromatin conformation capture, chromatin state annotation, DNase I hypersensitivity, hypomethylation, anatomical localization and biochronicity. We also made use of chromatin state data from the epigenome roadmap, transcription factor-binding data, spatial maps from published $\mathrm{Hi}$-C datasets and 'guilt by association' methods. Results: We identified 31 pharmacoepigenomic SNPs from a total of 2024 variants in linkage disequilibrium with lead SNPs, of which only $6 \%$ were coding variants. Interrogation of chromatin state using our workflow and the epigenome roadmap showed agreement on 34 of 35 tissue assignments to regulatory elements including enhancers and promoters. Loop boundary domains were inferred by association with CTCF (CCCTC-binding factor) and cohesin, suggesting proximity to topologically associating domain boundaries and enhancer clusters. Spatial interactions between enhancer-promoter pairs detected both known and previously unknown mechanisms. Addiction and analgesia SNPs were common in relevant populations and exhibited large effect sizes, whereas a SNP located in the promoter of the SLC1A2 gene exhibited a moderate effect size for lithium response in bipolar disorder in patients of European ancestry. SNPs associated with drug-induced organ injury were rare but exhibited the largest effect sizes, consistent with the published literature. Conclusion: This work demonstrates that an in silico bioinformatics-based approach using integrative analysis of a diversity of molecular and morphological data types can discover pharmacoepigenomic variants that are suitable candidates for further validation in cell lines, animal models and human clinical trials.
\end{abstract}

Keywords: chromatin spatial interactions $\bullet$ pharmacodynamics $\bullet$ pharmacogenomics

- pharmacokinetics

The majority of SNPs reported in genomewide association studies (GWAS) have examined drug response genes located in noncoding regions of the human genome [1,2]. However, in most association studies, emphasis has been placed on single gene variants that disrupt protein-coding domains because these have been identified as drivers of many inherited genetic disorders [3]. Increasing understanding of the $3 \mathrm{D}$ architecture of the human genome and its control by epigenetic markers both suggest mechanisms of action for noncoding variants and provide datasets to identify them. The importance of
Gerald A Higgins ${ }^{1,2}$ Ari Allyn-Feuer ${ }^{1}$ \& Brian D Athey ${ }^{*, 1,3}$

'Department of Computational Medicine \& Bioinformatics, University of Michigan Medical School, 1301 Catherine Road, Ann Arbor, MI 48109, USA

2Pharmacogenomic Science, Assurex Health, Inc., Mason, OH, USA ${ }^{3}$ Department of Psychiatry, University of Michigan Medical School, Ann Arbor, MI, USA

*Author for correspondence: bleu@umich.edu 
noncoding SNPs serving regulatory functions, such as enhancers, has been supported by the discovery of variants associated with psychiatric disorders such as schizophrenia [4], Type 1 diabetes [5], autoimmune disease [6], colon and other cancer risk [7,8] and a variety of other traits [9-15]. In addition, focus has been placed on the use of noncoding variants found in GWAS as components of related biological pathways [16]. Comprehensive application of these discoveries to optimized pharmacogenomic testing will bring us into a new era of pharmacoepigenomics.

Joint GWAS analyses of related disease phenotypes are now commonly accepted, as the evidence has mounted that overlapping sets of variants for related phenotypes are the norm, not the exception [17]. In addition, joint analysis of GWAS in psychiatric disorders using pathway analysis software has implicated shared, common pathways among multiple disorders such as bipolar disorder, major depressive disorder and schizophrenia [18]. Pathway analysis from multiple GWAS has been applied to recognize regulatory networks shared among common complex diseases, including coronary artery disease, rheumatoid arthritis, Type 1 diabetes, Type 2 diabetes and bipolar disorder [19].

Fine mapping of associated loci in GWAS yields causal variants that may differ from a reported lead SNP and are typically located in noncoding regions of the genome. For example, epigenetic mapping of enhancers using the histone marks $\mathrm{H} 3$ lysine 27 acetylation $(\mathrm{H} 3 \mathrm{~K} 27 \mathrm{ac})$ and $\mathrm{H} 3$ lysine 4 monomethylation (H3K4mel) in concert with mRNA-seq of neighboring genes was able to identify causal SNPs in enhancers in autoimmune disease, in which only $14 \%$ of associated protein-coding SNPs appear to be causal, whereas $60 \%$ of known causal SNPs map to enhancers [6]. The relative scarcity of missense coding SNPs in GWAS of disease risk and other human traits can now be simply explained: regulatory DNA harbors causal SNPs, in addition to protein-coding domains [20].

Research has illuminated the critical role that the $3 \mathrm{D}$ organization of the genome and epigenome plays in the regulation of gene expression. High-resolution $\mathrm{Hi}-\mathrm{C}$ demonstrates that the chromatin loop is the basis for regulation of gene transcription in the $3 \mathrm{D}$ context of the human genome, bringing together enhancers and gene promoters that may be located far apart in linear sequence [21]. Topologically associating domains (TADs) are fundamental units of transcription that may contain multiple chromatin loops, which bind enhancers to promoters with architectural proteins, including cohesin and CTCF [22,23]. DNA-dependent RNA polymerase II (POLR2A) is associated with both enhancers and promoters, and tissue-specific transcrip- tion factors are differentially enriched at enhancers or promoters [24].

The NIH Roadmap Epigenomics Mapping Consortium has enhanced our understanding of the role that the human epigenome plays in disease risk, phenotype and inheritance. Building on the foundation of the Encyclopedia of DNA Elements (ENCODE) consortium [24], the Roadmap Epigenomics project has elucidated features of the noncoding genome that contribute to interindividual variation in humans. The reference human epigenome [25] emphasizes the importance of histone marks that are indicative of active genome regulation (H3K27ac), active enhancers (H3K27ac and $\mathrm{H} 3 \mathrm{~K} 4 \mathrm{mel})$ and active promoters $(\mathrm{H} 3 \mathrm{~K} 27 \mathrm{ac}$ and $\mathrm{H} 3$ lysine 4 trimethylation [H3K4me3]).

In addition, to be accessible, noncoding SNPs that are associated with enhancers and other genome regulatory elements must be located in open chromatin that exhibits DNAse I hypersensitivity [26]. They are also hypomethylated, and may serve as quantitative trait loci [27]. Among the many important results produced by the Roadmap Epigenomics Mapping Consortium was not only the recognition that causal SNPs mined from GWAS are enriched in certain epigenome elements [6-7,28], but the analysis of haplotyped epigenomes, combining DNA-seq and Hi-C using HaploSeq [29], showed that variation in epigenomic state across all cell types and tissues within an individual was much less than that among individuals [30].

An ongoing challenge in pharmacogenomics is that many medications used in psychiatry and pain management are clinically suboptimal and exhibit high frequencies of serious adverse events. In pain management, opioids are highly addictive but very effective, so it would be better to develop analgesics without risk of abuse. However, the model compounds for almost all neuropsychiatric drugs were discovered in the 1950s or earlier, and included opiates, as well as chlorpromazine, imipramine, iproniazid and lithium. Because many medications used to treat patients with neuropsychiatric disorders were discovered over 50 years ago and/or serve as antecedents to later refinements of these seminal mid-century formularies, it is clear that greater emphasis should be placed on pharmacodynamic (PD) targets in the human CNS [31].

In this study, we developed a multilevel mapping method to identify novel noncoding SNPs in neuropsychiatry that exhibit epigenomic characteristics of genomic regulatory factors, and applied this method to screen SNPs derived from 26 pharmacogenomic association studies sourced from the NHGRI GWAS catalogue [32] and three published candidate gene-association studies. We used multiple known attributes of noncoding SNPs corresponding to genomic regulatory 
elements such as enhancers, promoters and transcribed domains using extant public resources. These included public databases of chromatin interaction mapping for prediction of cis- and trans-regulation, including Hi-C and ChIA-PET data [22,33-34], to predict putative spatial interactions at the allelic level. These interactions were also investigated for legitimacy based on 'guilt-byassociation' methods [35], leveraging multiple GWAS using pathway analysis $[16,17]$ and GWAS imputation and annotation methods published by the Roadmap Epigenomics Mapping Consortium [6-7,25,28,30,36]. At every phase of the experimental design, we used multiple independent analytic methods to ensure the validity of our in silico results using the current knowledge base.

\section{Methods}

The method and its components are shown in Figure 1 and described in the text. We used parallel approaches for SNP imputation, determination of functionally significant variants for a given pharmacogenomic association, annotation of SNPs and assignment to a genome regulatory element such as enhancer, promoter and transcribed element, prediction of putative functional interactions in cis and trans using chromatin interaction mapping data and novel pathway reconstruction using multiple methods, including assignment to the most plausible pathway in terms of known functional, pharmacologic and/or morphologic data. Most results were independently gathered by two investigators who were often not aware of the results obtained by the other. Because this study was performed in silico, several different, but sometimes related, methods were used to determine if the results were the same. The objective of this study was to provide evidence for subsequent experimental studies for scientific validation in cell lines, animal models and other biological systems.

\section{Examination of GWAS \& candidate gene-association studies}

We examined neuropsychiatric pharmacogenomic GWAS contained in the NHGRI GWAS catalogue [32] in psychiatry, neurology, analgesia and addiction, herein referred to collectively as 'neuropsychiatric.' These included 26 GWAS and three candidate geneassociation studies. The candidate gene studies were chosen for analysis because they were large case-control studies performed in circumscribed human populations that had identified significant variants associated with lithium response, citalopram response and heroin addiction, to demonstrate proof-of-concept. The gene-association studies used for the analysis are shown in Supplementary Table 1. Thirty-one variants from the 26 GWAS and three candidate gene-association studies were initially selected that reached the criterion of a pharmacoepigenomic SNP from a total of 2024 variants in linkage disequilibrium (LD) $>0.8$ $\left(\mathrm{r}^{2}\right)$ with the reported lead SNP within the population being measured. In total, 94\% (1903) of the 2024 SNPs were noncoding and 6\% (121) were coding.

\section{SNP imputation \& selection}

In certain cases, standard methods were used for dense genotyping and imputation from association loci that had not been performed in the original study, including removing spurious associations and checking population stratification $[6,37]$. In GWAS, if lead SNPs met the stringent statistical criterion of $\mathrm{p} \leq 5 \times 10^{-8}$, then the $\mathrm{SNP}(\mathrm{s})$ were checked for epigenomic classification using both our workflow (Supplementary Figures 1 \& 2) and the reference human epigenome [25]. In all but one case, the 31 SNPs analyzed using our workflow and the human reference epigenome produced the same results for the putative active regulatory elements that we identified. SNPs associated with mutations in an enhancer that controlled a subnetwork responsible for drug efficacy and adverse events were prioritized for further analysis. All gene definitions used in this study were acquired from the HUGO database [48]. Genes were mapped to UCSC Human Genome Browser hg19 coordinates [40]. Variation data from dbSNP [49] were also mapped to UCSC hg19 coordinates, and LD data for SNP pairs within population-specific haplotypes were downloaded from HaploReg [50]. After compiling a master spreadsheet containing pharmacoepigenomic SNPs as output of our workflow, we compared the results with those using the Roadmap EpiGenome Browser [28]. Although this study only focused on what may be functionally active genome regulatory elements, such as enhancers, promoters and transcribed domains, the Roadmap EpiGenome classification system includes 15 chromatin states. Nonetheless, for every variant that we characterized as a promoter, enhancer, transcribed domain or quiescent, 30 of 31 closely corresponded to the chromatin state annotated from the Roadmap EpiGenome. These data are presented in the 'Results' section.

In cases wherein dense genotyping and epigenetic fine mapping had not been performed in the original GWAS analysis, we used a simple derivation of the method developed by Farh et al. [6], with several modifications. We did not restrict GWAS selection to only those studies containing SNPs that met the rigid statistical criterion of $\mathrm{p}<5 \times 10^{-8}$. The rationale was that, as has been shown by others, SNPs that appear to be less significant may in fact contribute function or be causal, based on pathway analysis showing that the SNP may impact a genome regulatory element, such as an enhancer [5-6,12]. Distal H3K27ac peaks were assigned 
Examination of GWAS and candidate gene-association studies

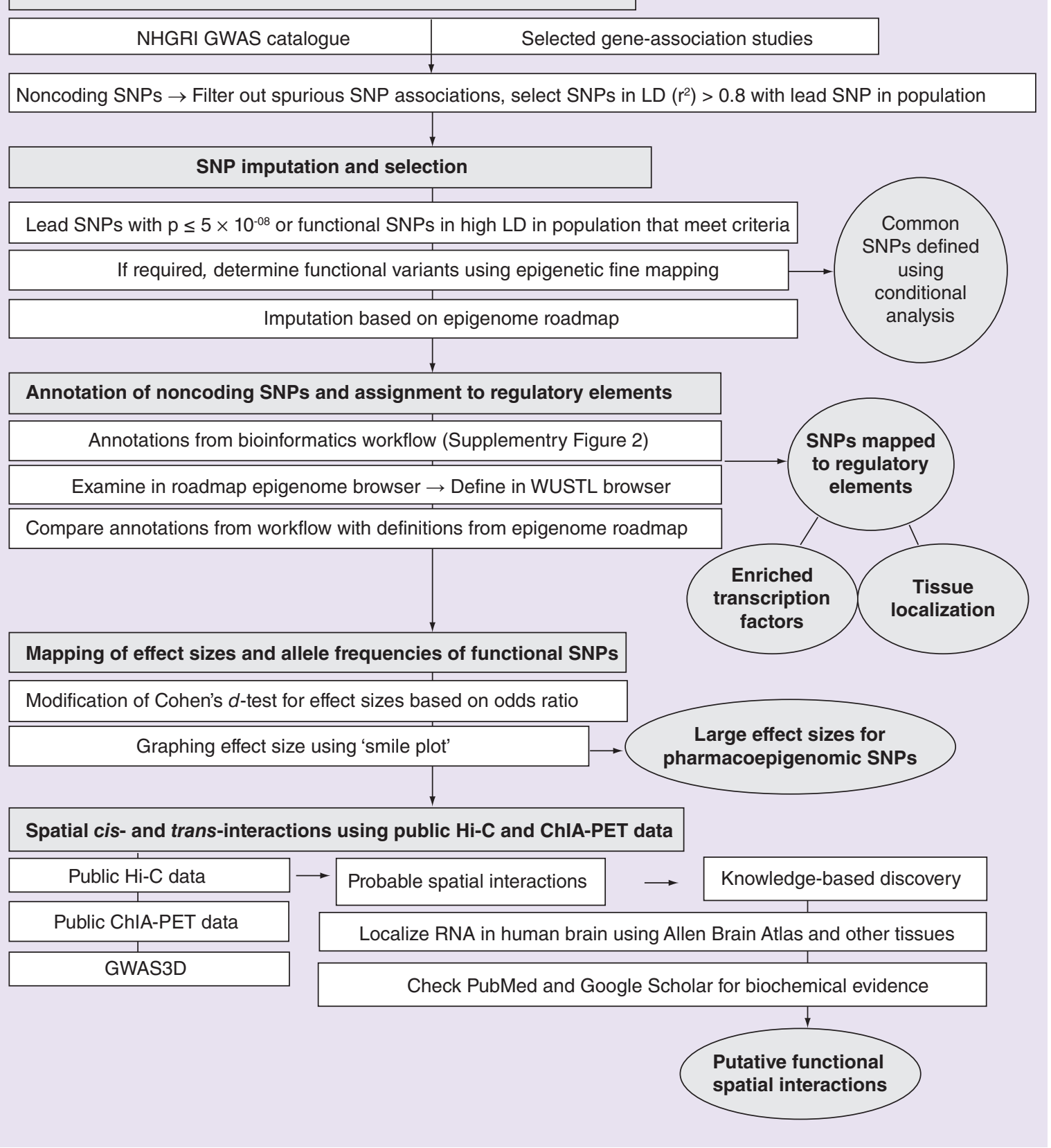

Figure 1. Overview of the methodology used in the study and results. Mapping of effect sizes and allele frequencies for functional variants. Headings in the 'Methods' section are shown in shaded rectangles, and the 'Results' section, in shaded spheres.

GWAS: Genome-wide association studies; LD: Linkage disequilibrium; NHGRI: National Human Genome Institute; WUSTL: Washington University in St Louis.

Data taken from $[6,22,25,28,33-34,37-47]$.

to their potential target genes if they were located within introns or within $500 \mathrm{~kb}$ regions upstream of a transcription start site of a gene that is likely a target of an enhancer. It is presumed that within an associated locus, a linear trend should be observed, such that where a causal variant has been demonstrated using functional studies, with the assumption that neutral SNPs demonstrate association signal in proportion to their LD to the causal variant. However, in GWAS, multiple causal SNPs that affect regulatory pathways may occur within a haplotype block with an $\operatorname{LD}\left(\mathrm{r}^{2}\right)$ $>0.8$ in the same population [6]. Conversely, a given 
locus may harbor causal SNPs associated with more then one phenotype. Thus, we did not discard what may have been considered neutral SNPs in the context of a pharmacogenomic association, but instead applied both statistical and knowledge-based methods to prioritize SNPs and identify them as functional, but not causal, in lieu of biological validation. If a reported lead SNP in an association study associated with a psychotropic drug response but was not correlated with a genome regulatory element expressed in human brain, nor associated with a pharmacokinetic $(\mathrm{PK})$ response, we examined all SNPs with an LD $>0.8$ in the haplotype of that population to determine if there were any in tight LD that exhibited correlation with human brain. This contributed to reprioritization of the variants in the LD block based on neuroanatomical localization. The methods used to identify functional SNPs in pharmacogenomic gene-association studies, and of genetic and epigenetic fine mapping for probabilistic identification of causal SNPs [6] are described in more detail in Supplementary Figure 2.

\section{Annotation of pharmacoepigenomic SNPs \\ \& assignment to regulatory elements}

As part of our workflow, we assigned SNPs to regulatory elements combining data from chromatin conformation capture, correlation with CTCF and cohesion (RAD21, SMC3) transcription factors associated with chromatin loop boundaries, chromatin state annotation, DNase I hypersensitivity, hypomethylation, anatomical localization and biochronicity. The method focused on variants, including SNPs associated with drug response and adverse drug events, which are used to identify genomic regulatory elements that are expressed in human brain or the liver in the case of a CYP gene, or regulate gene transcription, and are located in noncoding regions of the human genome. The workflow is comprised of several components, including determination of whether an epigenome variant may be functional, followed by its expression in brain (PD) or liver (PK).

Input variants are screened by ethnic population, followed by mapping of functional SNPs to determine the causal nature of a given SNP using methods based on the assumption that, in the majority of GWAS, it is necessary to discriminate SNPs that represent the most likely functional variants from those with strong but significantly less association to the drug response trait, so that most SNPs at a given locus can be excluded from further analysis. As a consequence of tight LD that exists within a haplotype or haplotypes associated with a trait, the lead $\mathrm{SNP}(s)$ as reported may or may not attain the threshold of significance used in GWAS and may not necessarily be the best candidate to serve as the functional or causal SNP.
The steps of the bioinformatics pipeline workflow in Supplementary Figure 1 were developed independently of the Roadmap Epigenomics Mapping Consortium. The method is optimized for association studies in pharmacogenomics in psychiatry, addiction medicine, pain medicine and neurology, herein referred to collectively as 'psychotropic' variants. The method focuses on variants, including SNPs associated with drug response and adverse drug events, which are used to identify genomic regulatory elements expressed in human brain or the liver in the case of a CYP gene, or to regulate gene transcription in PD pathways in human brain. RegulomeDB [27] provides an initial assessment as to what role a given variant might play in gene regulation. Variants are evaluated according to following criteria: whether they were enriched as quantitative trait loci (eQTLs, meQTLs) as determined using the scoring method of GeneVar [51] and/or GTEx [52]; whether the variant was located in open chromatin in the tissue of interest (e.g., brain, neuronal cell line, liver, immune cell, HepG2, heart or other); whether the variant was hypomethylated; and what specific histone marks were associated with the variant that defines its assignment as a promoter, enhancer or transcribed domain to impute chromatin state [36,53].

We also determined the degree to which a variant altered the strength, number and type of binding sites for transcription factors, and which transcription factors were bound to the variant to understand the function of the corresponding genomic regulatory element. These were determined using several public resources, including HaploReg 3.0 [50], and manual inspection of the variant in the UCSC Genome [40] and Roadmap EpiGenome [28] Browsers. Multilevel mapping in chromatin interaction networks for variants located in human brain help provide evidence for probable functional pathways. In neuropsychiatric pharmacogenomics, it is also critical to determine if annotated regulatory elements and their target genes exhibit biochronicity, a general feature of pharmacogenomic variation [54]. In the workflow analysis (Supplementary Figure 1), variants are assessed as to whether they are enriched as quantitative trait loci (eQTLs, meQTLs); whether the variant is located in open chromatin; whether the variant is hypomethylated; what specific histone marks are associated with the variant; the degree to which a variant alters the strength, number and type of binding sites for transcription factors; which transcription factors are bound to the variant; what interactions and/or contacts the variant makes with other genomic regulatory elements, such as transcription start sties (TSS) in chromatin networks; the location of the variant in brain regions or liver that are consistent with the function of the variant; and whether the variant exhibits biochronicity, a general feature of 
pharmacoepigenomic variation. These assessments can be performed using a variety of public bioinformatics resources. Other attributes may also be important, including evolutionary conservation as determined using GERP++, or whether a variant is within a human accelerated region of the genome. Biochronicity checking using published literature resources provides additional detail to the annotation model to determine if a given variant and its corresponding genomic regulatory element exhibit circadian, ultradian or seasonal rhythmicity. These signs are indicative of dysregulated core symptoms in the clinical context of psychiatric disorders such as bipolar disorder and major depressive disorder, and can be checked using the knowledge update engine. The impact, if any, of the glucocorticoid receptor (GR) on the variant is also checked, because it mediates the stress response and is the primary driver of circadian and ultradian rhythmicity. The input variant is also checked to determine if it is a member of the CLOCK gene family or a closely related gene.

We further examined the output of assignments from our workflow and compared them to those made by the Roadmap Epigenomics Mapping Consortium. This involved examination of the SNPs using the Roadmap EpiGenome Browser [28], followed by definition of regulatory elements using the WUSTL Genome Browser [38]. We then compared these results with the results shown in Figure 2 \& Supplementary Figure 3.

\section{Mapping of effect sizes \& allele frequencies of functional SNPs}

We calculated the effect size and associated allele frequency for a number of functional pharmacogenomic SNPs (those from studies reporting odds ratios), as well as a background set of SNPs associated with neuropsychiatric diseases. Minor allele frequencies were taken directly from published GWAS. Effect sizes were calculated using SNP frequencies and odds ratios using a variant on Cohen's $d$ [44]. These were plotted on a $2 \mathrm{D}$ smile plot (Figure 4) [45-46,55]. The plot also includes statistical power curves for reference. Power curves demarcating the lower bound for the effect size and frequency of SNPs detectable in a GWAS can be derived using the Package 'pwr' in $\mathrm{R}$ [56]. For reference we include the power curves of two hypothetical GWAS surveys, which would be only barely able to detect the median background SNP, for one curve, and the most powerful such SNP, for the second curve. SNPs above a given power curve are more significant, in a statistical sense, than those below and those on the curve. This plot provides a visual illustration of the comparative significance (in therapeutic terms) of a collection of SNPs based on their combined effect size and associated allele frequency.
Spatial cis- \& trans-interactions using public $\mathrm{Hi}-\mathrm{C} \&$ ChIA-PET data

For prediction of cis-interactions of putative enhancer, we examined genes that fell within the purview of known enhancers identified by the ENCODE and Roadmap Epigenomics Mapping consortia, and used knowledge-based prediction to identify those genes most likely to contribute to the drug response phenotype being evaluated. These were then plotted as shown in Figure 5. A combination of known enhancer lengths are shown above each of this subset of examples, with potential TAD boundaries represented as shown based on correlation of the SNP to boundary factors such as CTCF and cohesin (i.e., RAD21 and SMC3). We used several databases of Hi-C and ChIA-PET to identify enhancer-target gene interactions in cis and trans. These included public Hi-C datasets and ChIA-PET data visualized in the UCSC Genome Browser [22,39-40]. In addition, we used the GWAS3D website [33] to confirm some of the spatial interactions found in the primary datasets. The 3DGD database of Hi-C interactions in certain human cell lines was downloaded and provided additional value for discrimination of spatial interactions [34]. For cisregulation of gene promoters by enhancers, we used the most probable targets based on a 'guilt-by-association' method using known biochemical pathways and QTL relationships mined from published literature. In many cases of putative trans-interactions, target regions of the genome contained no known genes or lincRNAs whose specific function had not yet been understood. The Ensembl genome bowser [57] was used to evaluate enhancer targets because they provide the most comprehensive documentation of lincRNAs, as well as extensive tracks for predicted genes.

\section{Results}

Functional SNPs map to predicted pharmacoepigenomic enhancers

In this study, all of the predicted functional SNPs mapped to introns or intergenic regions, followed by $5^{\prime}$ UTRs and $3^{\prime}$ UTRs. Figure 2 shows an overview of the distribution of SNPs by genome region. Many intronic SNPs were associated with enhancers that did not apparently regulate the gene in which they were located. Next, we compared the regulatory element annotation from the Roadmap EpiGenome Browser with the results of our workflow (Figure 2 \& Supplementary Figure 3). The assignments between the two independent methods was high, with concordance in regulatory element class in different adult human tissues for 34 of 35 assignments. Comparison with ENCODE cell line annotations also showed $97 \%$ agreement with our workflow (data not shown). 

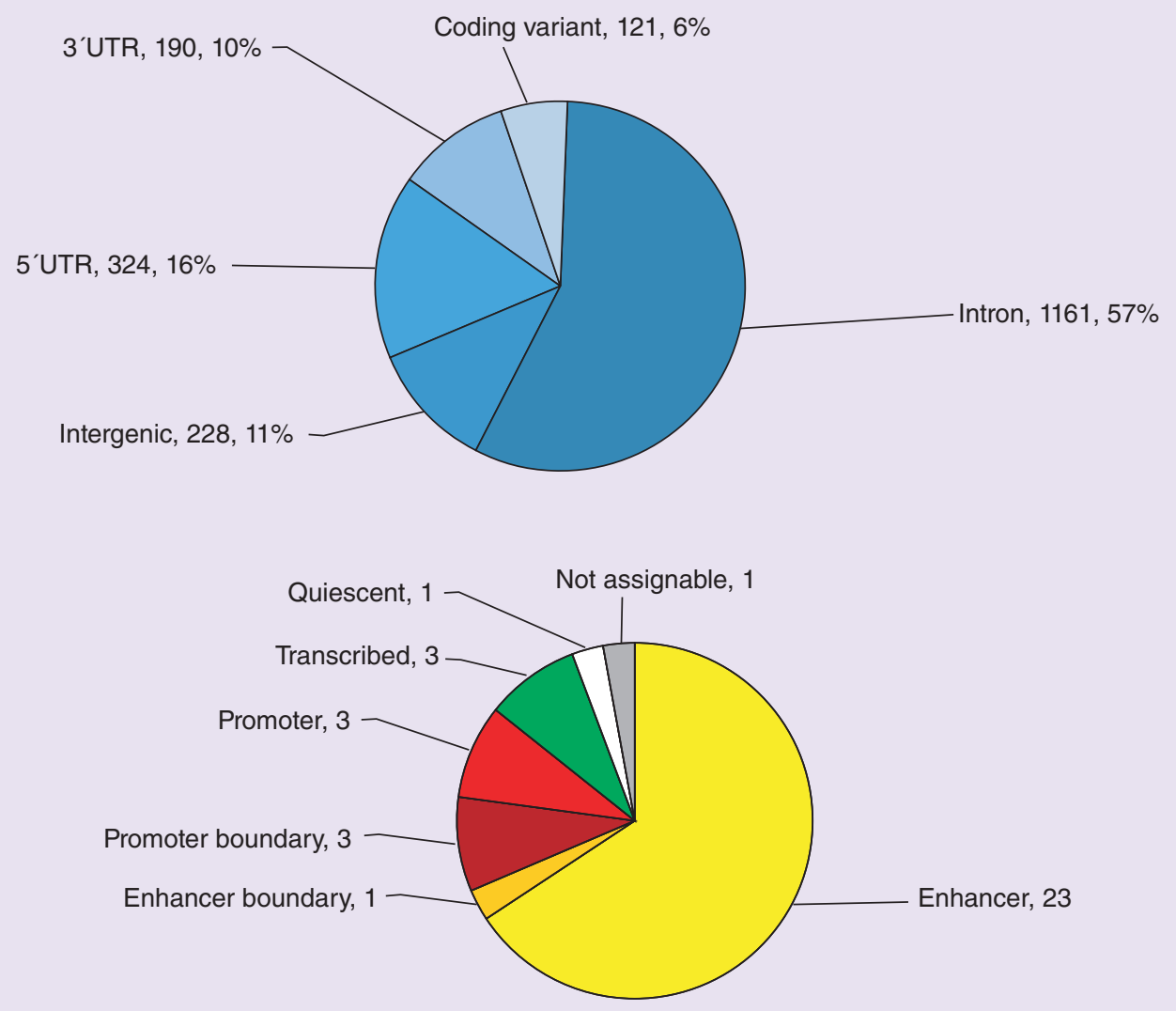

Figure 2. Assignment of noncoding SNPs to regulatory state. Pharmacogenomic SNPs from genome-wide association studies in neuropsychiatry map to noncoding regions of the genome.

Since we were including association with CTCF and cohesin (subunits RAD21 and SMC3) to define location within enhancers and promoters near presumptive borders between loop domains, we have defined a new category of chromatin states which are loop boundaryrelated. As seen in Figure 2, tissue-specific assignments included 23 enhancers, one enhancer boundary, three promoters, three promoter boundaries, three transcribed domains, one quiescent domain and one that was not assignable.

\section{Enrichment of transcription factors at putative enhancer, promoters \& transcribed domains}

Previous research has shown that transcription factors and DNA-binding proteins bind in a sequence specific manner. Since the majority of our predicted functional SNPs are located in regulatory sequences in the relevant tissues (PD genes in brain and PK genes in liver), we used this knowledge to classify regulatort elements using annotation with DNA-binding proteins. Thus, EP300 is associated with enhancers, YY1 is associated with promoters, and the splicing factor TCERG1 is associated with transcribed domains. We selected those transcription factors associated with various regulatory elements and tissue type. Figure 3 plots some of the known transcription factors and other DNA-binding proteins whose binding was predicted to be altered by the SNPs, bound using ChIP-seq, or both, and were indicative of assignment to specific classes of regulatory elements. Statistical analysis showed that these specific transcription factors and DNA-binding proteins were associated with the consensus assignment to class of regulatory element $(\mathrm{p}<0.01$; ANOVA).

\section{Tissue-specific localization of regulatory elements}

Tissue localization of regulatory elements was mapped based on data from the epigenome roadmap consortia [28] and is shown in Supplementary Figure 4. Localization based on chromatin state annotation was in most cases consistent with organs involved in GWAS phenotypes, with HLA allele SNPs the main exception. The majority of regulatory elements were localized in liver and brain, consistent with a role in PK 


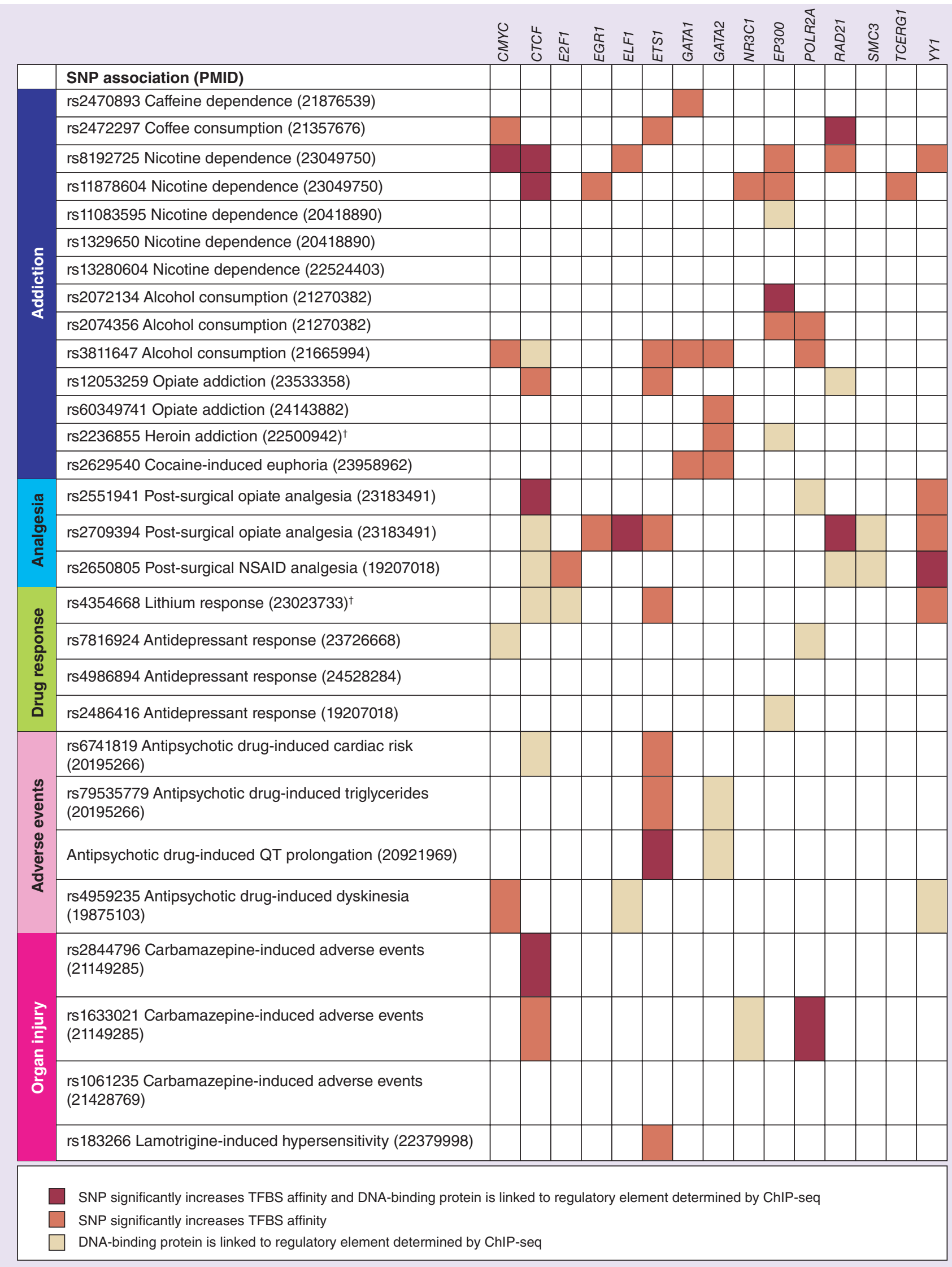


Figure 3. Association of transcriptional factors and other DNA-binding proteins helps define the domain occupied by different regulatory elements that could be unequivocally assigned and are active (see facing page). CTCF and cohesion subunits (RAD21, SMC3) are significantly associated with enhancer and promoter boundary domains. CYMC, E2F1, ELF1, ETS1, GATA1, GATA2 and EP300 are associated with enhancers. EGR1 is associated with estrogen regulation, and NR3C1 association is suggestive of glucocorticoid regulation. TCEGR1 is a transcriptional elongation factor associated with differential splicing. $Y Y 1$ is associated with promoters.

${ }^{\dagger}$ Published gene-association studies.

and PD pathways, respectively. Also, in GWAS of adverse events from antipsychotic medications, the pattern of expression is consistent with affected organs. These include perphenazine-induced cardiac risk in which the enhancer is expressed in brain and heart, and clozapine-induced elevated triglycerides, wherein the impacted enhancer is expressed in brain and pancreas. In carbamazepine- and lamotrigine-induced adverse events, the predicted enhancer was localized in CD34 or CD14 cells. Although very specific liver and brain-specific transcription factor clusters have been described [30], we were only able to characterize three examples in which tissue-specific transcription clusters could be assigned (Supplementary Figure 4).

\section{Large effect sizes for SNPs associated with} addiction, analgesia \& drug-induced injury In contrast to the prevailing understanding that complex traits are predominantly influenced by many variants with small effects, we found that causal SNPs in neuropsychiatric pharmacogenomics GWAS typically exhibit large effect sizes. Figure 4 shows a smile plot comparing allele frequency from 0 to 1 versus effect size calculated using a revision of Cohen's $d$ based on odds ratio [44-47,55]. As can be seen from the plot, functional pharmacoepigenomic SNPs associated with caffeine, nicotine, alcohol and opiate addiction are common within human populations and exhibit large effect sizes. Similarly, HLA allele tagging SNPs for carbamazepine and lamotrigine, medications used to treat bipolar disorder, exhibit very large effect sizes but minor allele frequencies. SNPs associated with lithium response and antipsychotic drug-induced cardiac risk are also common and have moderate effect sizes. Effect sizes for functional SNPs reported in psychotropic pharmacogenomic association studies were substantially larger than those reported in GWAS examining disease-risk variants for psychiatric and neurological disorders (Figure 4 \& Supplementary Table 2).

\section{Knowledge-based discovery of putative spatial interactions in cis \& trans}

Multiple public datasets were used to evaluate the spatial interactions of enhancers and promoters impacted by SNPs in gene-association studies of psychotropic pharmacogenomic phenotypes, followed by knowledge-based discovery methods using published literature as described. Spatial interactions were plotted as shown in Figures 5 \& 6 . Several functionally important relationships were inferred based on systematic evaluation of published literature, as well as other data sources such as ClinicalTrials.gov [58]. These relationships are concordant with RNA-Seq results [28]. In some cases, the association of enhancer RNAs (eRNAs) with this subset of known enhancers supported their role as active genome regulatory elements in the tissue of interest.

Figure 5 shows examples of SNPs that disrupt enhancers associated with addiction and analgesia. The SNPs rs2470893 and rs2472297 are well-replicated, statistically significant associations with caffeine dependence in European populations [59,60]. In cis, not only does the cognate enhancer control CYP1A1 gene expression, but also the regulation of the CYP1A2 gene, whose product is the rate-limiting enzyme for the metabolism of caffeine (Figure 5A) [61]. In addition, the large enhancer appears to regulate the $N K X 2-5$ gene, in which a SNP is significantly associated and correlated with increased caffeine metabolism in the liver [62]. For long-distance (trans) interactions, the identified enhancer disrupted by these SNPs is in spatial contact with the CACNA1B gene, as determined by $\mathrm{Hi}-\mathrm{C}$ in immortalized liver cells (HepG2; Figure 5A). Mutations in the CACNA1B gene have been linked to caffeine-induced anxiety in animal models [63]. In a study of nicotine dependence in a Japanese population [64], the statistically significant lead SNP rs11878604 disrupts an enhancer that regulates expression of the CYP2A6 gene, which encodes a protein that metabolizes nicotine to cotine [61], as well as $C Y P 2 A 13$, whose product $\mathrm{CP} 2 \mathrm{AD}$ metabolizes the major nitrosamine in tobacco, 4-(methylnitrosamino)1-(3-pyridyl)-1-butanone (Figure 5B) [65]. An enhancer involved in nicotine dependence [66] is linked in trans to the promoter of the CHRNB2 gene, in which variants are correlated with the euphoric effects of nicotine [67] (Figure 5B) and the SNP rs11083593, located in the same locus, may activate differential splicing of the $C Y P 2 B 6$ gene through a long-distance interaction with the TCERG1 gene that generates a splice variant associated with increased incidence of lung cancer (Figure 5C) [68]. The SLC17A8 gene is a glutamate transporter in brain whose expression has been significantly associated with addictive behaviors [69]. The SNP rs2336855, which is located in an intron of the OPDR1 gene, is associated with a population of individuals of European ancestry who were heroin addicts 


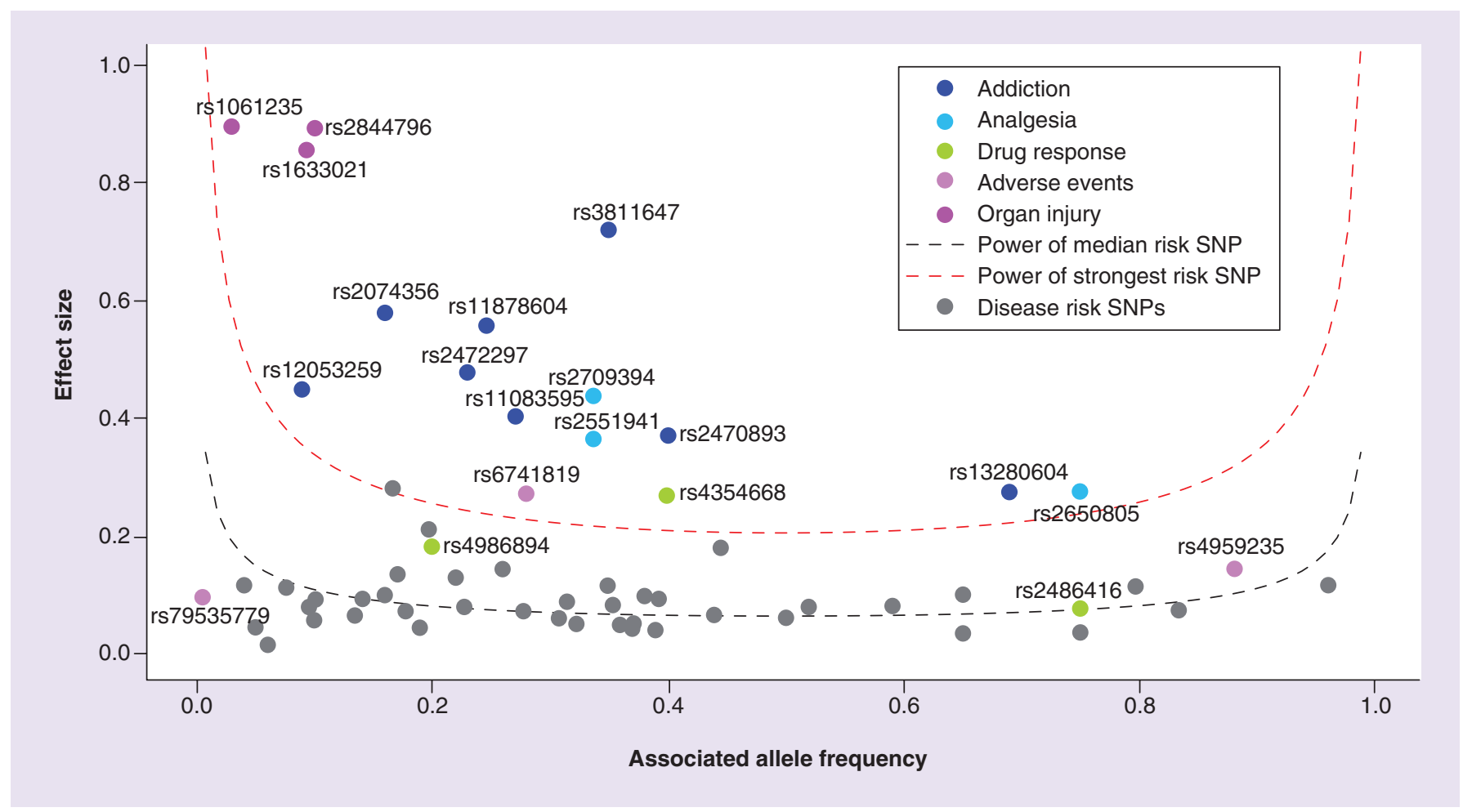

Figure 4. In contrast to neuropsychiatric disease risk SNPs, which characteristically have low effect sizes, psychotropic drug SNPs (colors) exhibit moderate-to-large effect sizes and moderate-to-high allele frequencies. Almost all exceed the statistical power threshold of the median risk disease SNP (gray dotted line) and $80 \%$ exceed the power of all disease SNPs (red dotted line). Noncoding SNPs associated with addiction (dark blue) and analgesia (light blue) are notable for large effect and high frequency. Tagging SNPs at HLA loci associated with drug-induced organ injury (magenta) exhibit very high effect sizes but rarer allele frequencies. The SNP rs4354668 associated with lithium response in bipolar I patients of European ancestry (green) exhibits a moderate effect size and a common allele frequency, as does SNP rs6741819 (violet), which is associated with antipsychotic druginduced cardiac risk. Neuropsychiatric disease risk SNPs were mined from the NHGRI GWAS catalog for bipolar disorder, schizophrenia, major depressive disorder, migraine and epilepsy and are indicated in gray. See Supplementary Table 2 for details.

in a large case-control candidate study that has been replicated (Figure 5D) [70]. The same enhancer appears to control the splicing factor SRSF4, which is thought to be responsible for alternative splicing of the OPRD1 gene [71]. In trans, it contacts the GABRA3 gene, in which variants associated with reward deficiency and opiate addiction have been identified (Figure 5D) [72,73]. In a GWAS of opioid dependence [74], a significant SNP imputed from a population of African-American males shows that the enhancer regulates NCK2, which encodes a protein involved in synaptogenesis, and has been shown to be significantly associated with morphine tolerance in an animal model (Figure 5E) [75]. The SNP rs2074356, which is significantly associated with alcohol dependence in a Korean population [76], impacts an enhancer that modulates the $A L D H 2$ gene, in which variants are significantly associated with alcohol-related neuropathy and cancer (Figure 5F) [77,78]. The enhancer also effects the CTSB gene, which has been correlated with addictive behavior in humans, alcoholism and alcohol-induced liver injury [79-81]. The enhancer is in spatial contact with the promoter of the ATNX2 gene on the same chromosome. Variants in the ATXN2 gene are responsible for a disorder called spinocerebellar ataxia [82], which is a characteristic adverse event associated with alcohol intoxication (Figure 5F) [83].

Figure 6 shows examples of the spatial interactions of enhancers and a promoter associated with psychotropic drug response and enhancers associated with antipsychotic drug-induced adverse events. These spatial relationships suggest the nature of the interaction, which provides insight into the mechanism or components of the potential pathway. In a GWAS of sustained antidepressant response, the significant SNP rs7816924, which is located in an intron of the CSGALNACT1 gene, contacts the CNTNAP3 gene in trans. CNTNAP3 encodes a contactin-associated protein located at central synapses in which variants have been significantly associated with autism, intellectual disability syndromes and depression (Figure 6A) [8487]. This enhancer also appears to regulate the DLC1 gene, whose expression in human brain, methylation state and variants have been significantly asso- 
ciated with posttraumatic stress disorder, substance abuse, suicide attempts and suicidal ideation [88-90]. The SNP 4354668 located in the promoter of the SLC1A2 gene, which encodes a high-affinity glutamate transporter in human brain, is correlated with lithium response [91], exhibits a large effect size in patients of European ancestry with bipolar disorder (Figure 4), and is connected in trans to the PLD5 gene (Figure 6B). Variants in the PLD5 gene have been significantly associated with bipolar disorder in patients of European ancestry [92]. Mutations in the SLC1A2 gene have been significantly linked to essential tremor in GWAS; tremor being a significant side effect of lithium treatment [93]. In a GWAS for monitoring the adverse event of QT prolongation in patients who take second-generation atypical antipsychotic medications [94], the imputed SNP rs4959235 is associated with quetiapine-induced QT prolongation, and the same enhancer impacts NQO2 gene in cis, which has been associated with clozapine-induced agranulocytosis [95], and is involved in acetaminophen hepatotoxicity (Figure 6C) [96]. This enhancer is spatially connected with the NOSIAP gene, which has been shown to control QT prolongation in multiple, replicated studies (Figure 6C) [97-100]. In a GWAS examining the risk for cardiovascular disease as a consequence of antipsychotic medication use in a European population [101], the corresponding enhancer appears to regulate the RNF144A gene, in which variants have been significantly associated with cardiac dysfunction (Figure 6D) [102].

\section{Discussion}

This study constitutes an in silico approach for identification of candidate SNPs that may provide value in future clinical studies for stratification of drug response phenotype. It is limited in that predictions are based on extant GWAS and gene-association data without accompanying biological validation. Nonetheless, discovery of regulatory variants that predict clinically important phenotypes has potential value. This study was undertaken to determine if candidate pharmacoepigenomic regulatory variants in humans could be identified using informatics and extant public data resources prior to further analysis in cell lines, animal models and clinical trials. Although these are bioinformatics-based predictions, they differ from genome-wide applications such as ChromHMM [53], ChromImpute [25], GBR [103] and Segway [104] because they make use of context-specific datasets collected in disease-relevant tissue types. For this reason, and because they are derived from geneassociation studies in human populations and many exhibit substantial effect sizes, our candidate variants and their predicted sites of action represent candidates for subsequent investigation and use in biological experiments, biomedical and clinical validation research and, ultimately, in clinical practice. Like any new approach in biomedical research that needs further experimental validation, epigenomic analysis of noncoding variants that explain gene regulation will demonstrate its utility through a more thorough understanding of the corresponding phenotype. We note that many of the new intragenic and intronic pharmacoepigenomic SNPs we have uncovered in this work are of the PD class, which is under-represented in neuropsychiatric pharmacogenomics today.

The informatics-based predictions of mechanisms found in this study, as illustrated in Figure 5 \& Figure 6, can be validated by subsequent experimental studies in cells and tissues to observe spatial interactions between promoter-enhancer pairs in cell lines and tissues. These studies may use methods such as FISH microscopy, tissue-based $\mathrm{Hi}-\mathrm{C}$ and related methods for the study of dynamic genome architecture as was demonstrated in Rao et al. [21]. These variants and others found using these approaches may also be considered high priority variants for combinatoric effect size analysis. This analysis provides a straightforward and comprehensive approach to prioritizing psychotropic pharmacoepigenomic variants and corresponding regulatory pathways that will support a new generation of clinical pharmacogenomic tests and CNS drug discovery.

In addition, these results and others point to an emerging understanding about potential new ways to interpret GWAS datasets.

First, the importance of noncoding regulatory variation in the determination of pharmacogenomic response and adverse drug events is now being realized. Although nonsynonymous mutations within exons contribute to disease risk and variable drug response, this biological phenomenon cannot begin to explain the human variome, with additional clarity provided by epistasis and epigenomics. Zanger et al. [105] have discussed this phenomena concerning noncoding SNPs in CYP genes. Because significant evidence suggests that most variation in human traits lies outside protein-coding regions of the genome [20], epigenome informatics provides a step toward a better understanding of the variation in pharmacogenomic traits that exist in noncoding regions of the human genome [27,106].

Second, the epigenome, in addition to serving as a mechanism for nonsequence inheritance and regulation of transcription patterns, is important in both clarifying which variants are important and predicting their mechanism of action. The brain generates the highest 
(A)

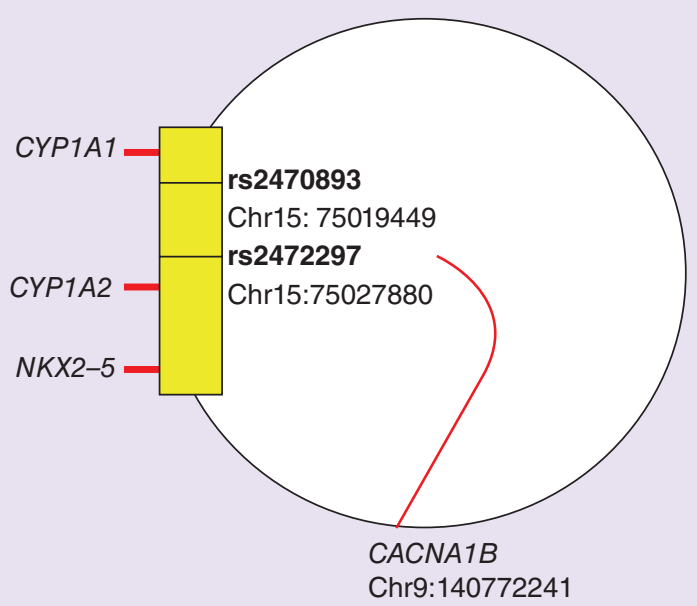

(C)

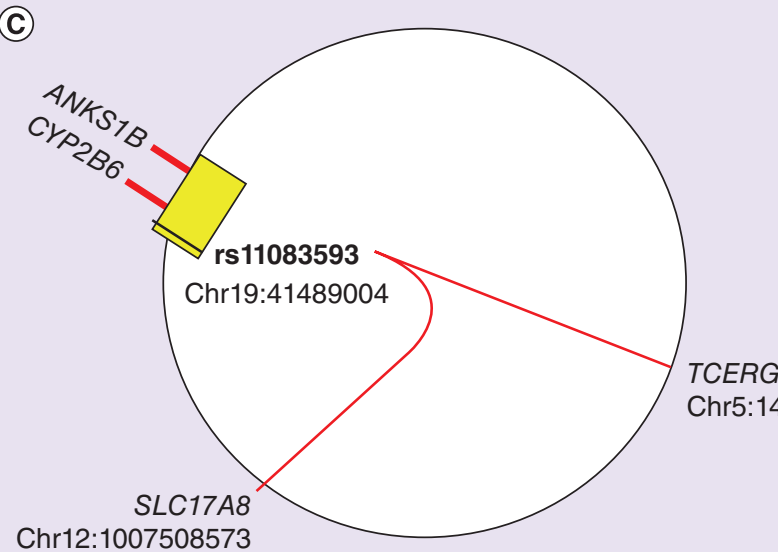

(E)

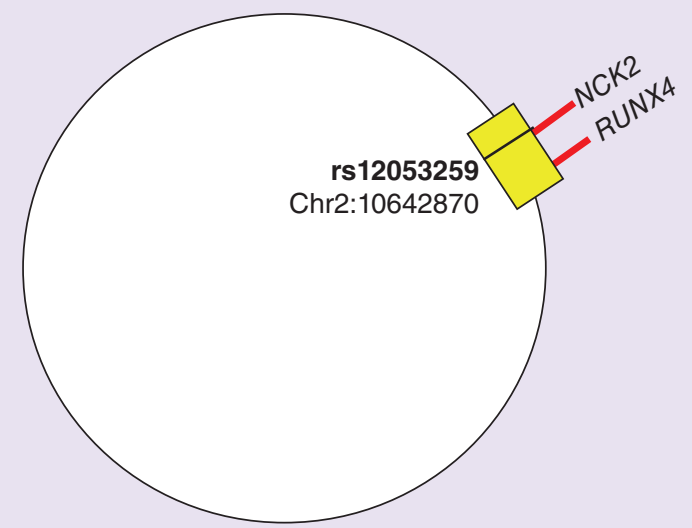

(B)

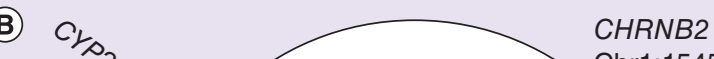

Chr1:154540257

(D)
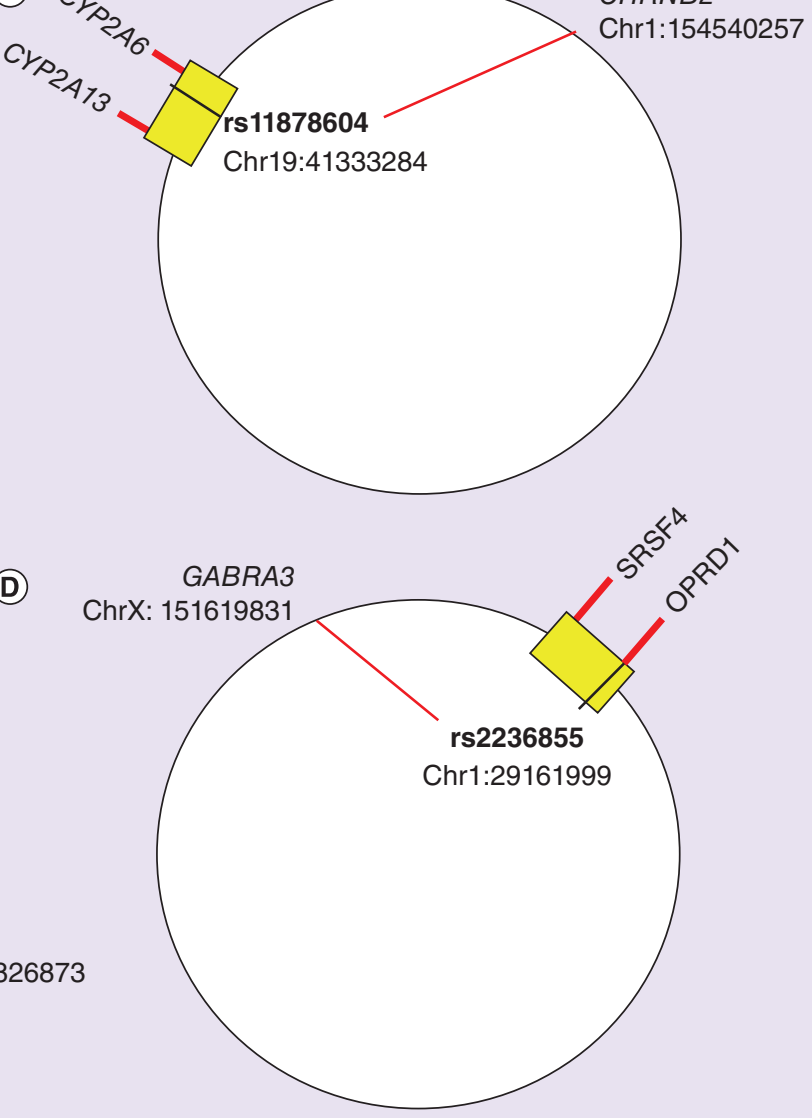

(F)
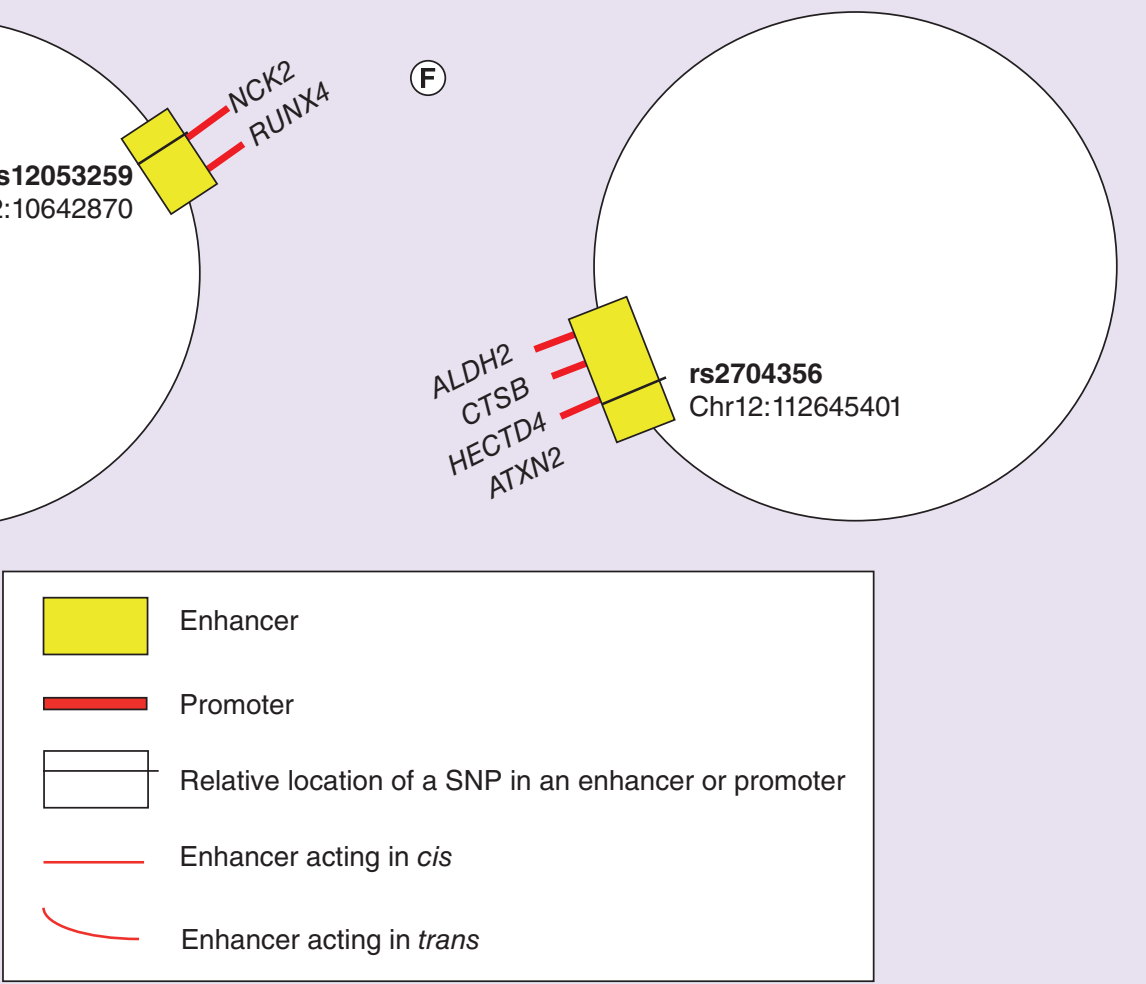
Figure 5. Spatial interactions of enhancers associated with addiction and analgesia plotted on circular genome-wide maps (see facing page). (A) SNPs significantly associated with caffeine dependence in individuals of European ancestry replicated in multiple GWAS; (B) a SNP significantly associated with nicotine dependence in a Japanese population; (C) a functional SNP associated with heroin addiction in Europeans; (D) a SNP associated with opioid addiction in African-American males; (E) a SNP significantly associated with alcohol dependence in a Korean population; (F) This SNP impacts an enhancer significantly associated with alcohol abuse in a Korean population.

transcriptional complexity of any tissue by more than an order of magnitude [107,108], involving transcription of at least $40 \%$ of all human coding genes [25], and the regulatory networks involved in neuropsychiatric diseases are likely to be similarly complex. Thus, it is prob-

able that the spatial connections of the enhancers, promoters and transcriptional elements detected by these significant variants can provide explanatory value, given the examples shown in Figures 5 \& 6 . New data types, including haQTLs, pQTLs, metQTLs and sequence-
(A)

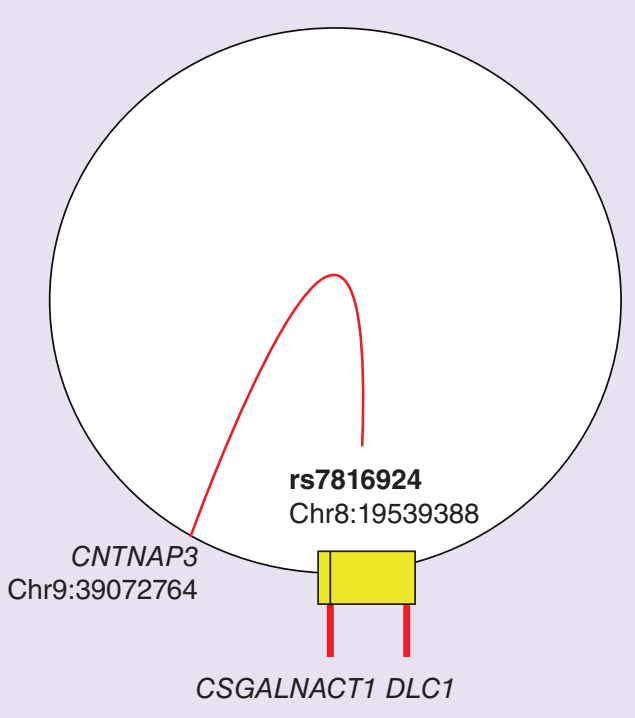

(C)

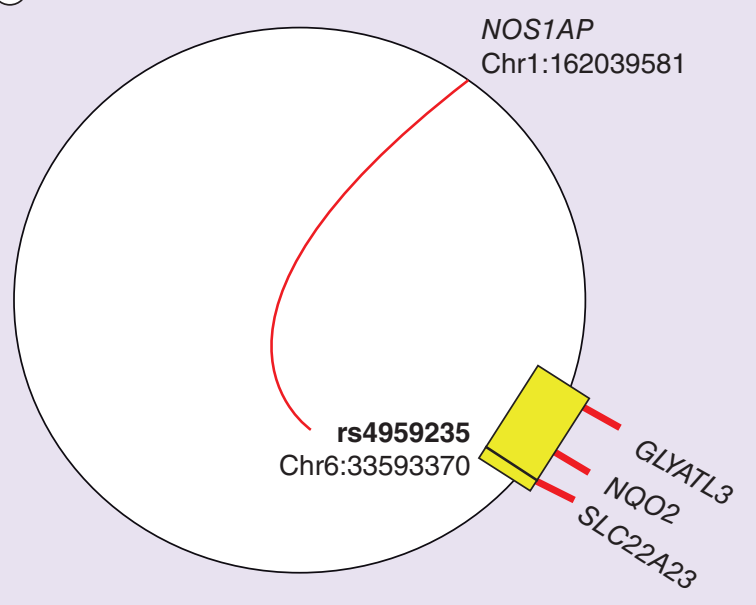

(B)

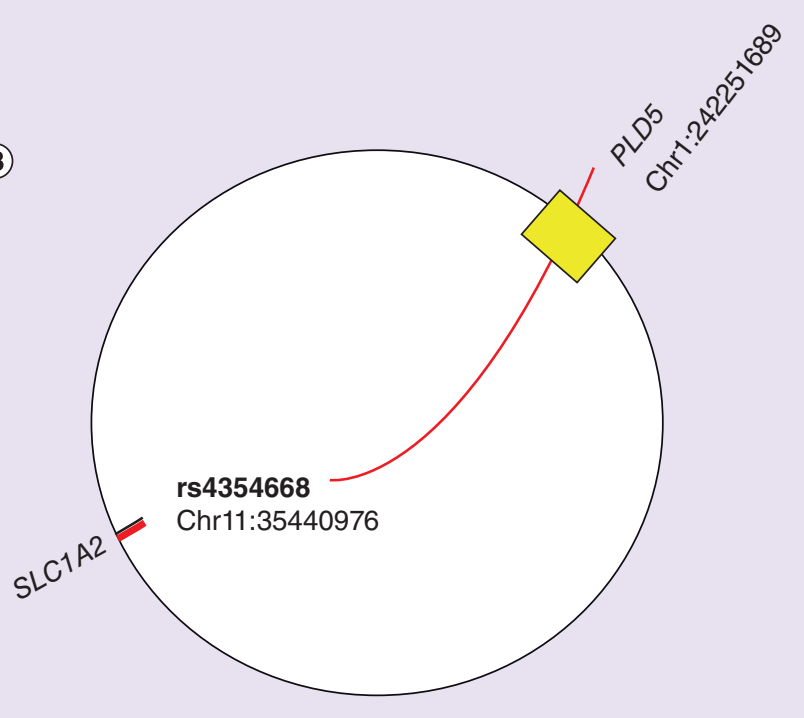

(D)

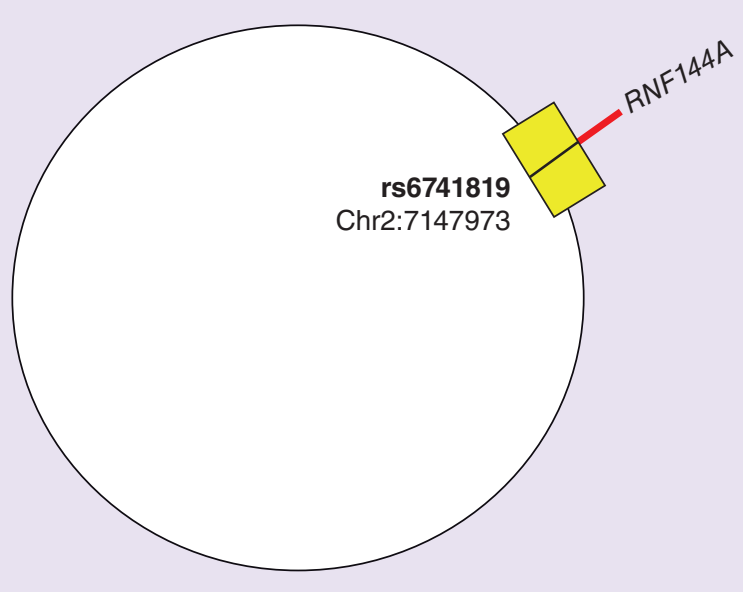

Figure 6. Spatial interactions of enhancers associated with psychotropic drug response and antipsychotic-induced adverse events plotted on circular genome-wide maps. (A) A SNP associated with sustained antidepressant response; (B) a SNP located in the promoter of the SLC1A2 gene associated with lithium response in bipolar patients of European ancestry; (C) a functional SNP associated with quetiapine-induced prolonged QT syndrome in an American population; (D) A SNP associated with antipsychotic drug-induced cardiac risk in a European population. For tissue localization, refer to Supplementary Figure 4. 
based predictions of variant effects on the epigenome, will probably also provide explanatory value.

Third, the 4D nuclear context (3D structure plus temporal dynamics) is quite important in interpreting neuropsychiatric GWAS. In parallel with emerging results from studies of $3 \mathrm{D}$ nuclear architecture, phenomena such as circadian rhythmicity of gene expression [54] and the timing of interactions between specific CLOCK gene loci demonstrated by $\mathrm{Hi}-\mathrm{C}$ [109] emphasize the importance of temporal dynamics for understanding the functional impact of gene expression. New evidence is challenging existing dogma, including recognition of the not uncommon nature of monoallelic transcription in mammalian cells as determined using single cell RNA-seq and 3D FISH at the cellular level [110-113] and allele-specific expression at the tissue and population level, distinct from known mechanisms of genomic imprinting [114,115]. Noncoding variants identified in GWAS can be annotated as genomic regulatory elements [50,106], supporting the discovery of novel regulatory pathways that can be studied temporally.

Fourth, GWAS have demonstrated variable utility in different systems. GWAS research in complex human diseases has frequently shown a large number of small-effect, high-frequency SNPs (Figure 4) [45,55], and seldom shown common high-effect SNPs [116]. Our results clearly demonstrate that neuropsychiatric pharmacogenomic phenotypes do not exhibit the same constraints. Several of the pharmacogenomic systems we investigated display SNPs that are sufficiently powerful and in high frequency such that, alone or in combination [117], they could potentially be used in clinical pharmacogenomic assays if validated in clinical research studies.

The reasons why this appears to be common in pharmacogenomics but rare in disease prediction remain unclear. The literature in disease GWAS is converging on the notion of an evolutionary 'ceiling' on the significance (prevalence and effect size) of individual variants imposed by the deleterious fitness effect of disease $[44,46]$. Although it could be argued that pharmacogenomic phenotypes would not be subject to the same evolutionary constraints because some of these medications only appeared recently in evolutionary terms, this explanation makes less sense in light of the relatively large effect sizes observed for SNPs for naturally occurring addictive drugs such as alcohol and opiates which have been used by humans for at least 3500 years (opiates) to 9000 years (alcohol) $[118,119]$. Further work will define the contours of this phenomenon, but it is clear that the utility of GWAS may vary substantially by phenotypic class, rendering global judgements in this area premature.
Fifth, the relevance of the traditional significance threshold (and in the future, any hard threshold) is declining. While the Bonferroni or ' $\mathrm{X}$ ') threshold often limits the number of significant lead SNPs reported in pharmacogenomic studies, recent studies have used imputation methods to identify variants in high LD with sub-Bonferroni lead SNPs, often greatly increasing the significance of functional or causal variants that represent bona fide associations with the trait of interest as opposed to so-called tagging SNPs [120]. Other researchers have convincingly argued that more informed systems approaches indicative of pleiotropy, including false discovery rate, are more accurate measures of significance in GWAS compared with traditional metrics [121-123]. The totality of these strategies and our findings demonstrate that there is a great deal of useful data to be mined from existing GWAS that may have previously been dismissed as inconsequential [124]. There is every indication that a strategy of permissive thresholding, followed by screening with other forms of information, is to be preferred, at least in further pharmacogenomic studies. In the future, 'co-imputation' strategies may emerge which dispense with GWAS significance thresholds entirely and screen variants with a combination of association and other methods from the very beginning of the workflow.

The results presented here are promising targets for future research that validate neuropsychiatric pharmacogenomic regulatory variants as clinical biomarkers, and the methods used to discover them, as part of an emerging new understanding of GWAS methods and significance, can offer powerful utility for discovering, interpreting and validating functional variants in a number of systems, eventually adding to the considerable value already obtained from pharmacogenomic testing. Such variants may include not just SNPs but also indels, and these methods are extensible to probe variants called from NGS profiles of patient cohorts. Results from this study provide insight into potential regulatory mechanisms of gene expression underlying psychotropic drug responses, and establish an in silico bioinformaticsbased pipeline approach to discover novel putative pharmacoepigenomic variants. This approach can serve to streamline the discovery of novel variants, which can then be further validated in cell lines, animal models and clinical trials using a diverse set of molecular and morphological data types.

\section{Supplementary data}

To view the supplementary data that accompany this paper please visit the journal website at: www.futuremedicine. com/doi/full/10.2217/PGS.15.105 
Financial \& competing interests disclosure

GA Higgins is an employee of Assurex Health, Inc. (Mason, $\mathrm{OH}$, USA) and holds options in the company. He serves as an Adjunct Research Professor at the University of Michigan (U-M) Medical School. Assurex Health, Inc. and the University of Michigan have established a Master Research Agreement under which this work falls. GA Higgins has a Conflict of Interest Management Plan on file at U-M. A Allyn-Feuer is a Bioinformatics Graduate Student and was funded by NIH NIGMS U-M Bioinformatics Training Grant T32 GM0704490552 (BD Athey, PI) and U-M research funding. A Allyn-Feuer has no conflict of Interest. BD Athey is chairman of the Department of Computational Medicine and Bioinformatics, University of Michigan Medical School. He also serves as Chair of the Scientific Advisory Board of Assurex Health, Inc. and holds options in the company. He has performed this work as a University of Michigan Professor. Assurex Health, Inc. and the University of Michigan have established a Master Research Agreement and a Conflict of Interest Management Plan for BD Athey. The authors have no other relevant affiliations or financial involvement with any organization or entity with a financial interest in or financial conflict with the subject matter or materials discussed in the manuscript apart from those disclosed.

No writing assistance was utilized in the production of this manuscript.

\section{Ethical conduct of research}

The authors state that they have obtained appropriate institutional review board approval or have followed the principles outlined in the Declaration of Helsinki for all human or animal experimental investigations. In addition, for investigations involving human subjects, informed consent has been obtained from the participants involved.

\section{Open access}

This article is distributed under the terms of the Creative Commons Attribution License 4.0 which permits any use, distribution, and reproduction in any medium, provided the original author(s) and the source are credited. To view a copy of the license, visit http://creativecommons.org/licenses/by/4.0/

\section{Executive summary}

- Analysis of genome-wide association studies (GWAS) data is involving increasing emphasis on noncoding variants, joint analysis of related phenotypes and fine mapping of linkage disequilibrium regions.

- Recent research has revealed the key role of the epigenome and 3D nuclear organization in regulation of gene expression.

- Neuropsychiatric drugs have serious problems with both response and adverse events.

- We propose to use these advanced GWAS methods, the epigenome and the 4D nucleus, to examine neuropsychiatric drug phenotypes.

\section{Methods}

- We examined 26 GWAS and three candidate drug association studies in psychiatry, neurology analgesia and addiction.

- Resulting SNPs were screened using an integrative flow with many forms of genetic and epigenetic information including methylation, chromatin modification, transcription factor (TF) binding, sequence context, $\mathrm{Hi}-\mathrm{C}$, gene expression data and biochronicity data.

- The high-quality candidates were annotated to chromatin states using both our workflow and the Roadmap Epigenome ChromHMM data.

\section{Results}

- The predicted functional SNPs mapped to promoters and enhancers, not coding regions.

- They were enriched for TF-binding motifs modified by variants, and for measured binding of those same TFs in relevant tissues.

- They were highly enriched at predicted promoter and enhancer regulatory regions, and our workflow had $97 \%$ concordance with the epigenome roadmap in identifying such regions.

- Regulatory element calls were tissue-specific to relevant tissues.

- Our candidate variants exhibited a combined effect size and allele frequency in excess of that observed for disease risk. Eighty percent exceeded the statistical power of all neuropsychiatric disease variants.

\section{Conclusion}

- Noncoding variants appear to be causative in many phenotypes.

- The epigenome can both clarify which variants are important and predict their mechanism of action.

- The 4D nuclear context (3D structure plus temporal dynamics) is important in interpreting neuropsychiatric GWAS because of the pervasive temporal transcriptional complexity in the brain.

- GWAS as a strategy is of variable utility in different systems; however, pharmacogenomics may yield promising results for further research.

- Our overall approach can serve to streamline the discovery of novel variants, which can then be further validated in cell lines, animal models and clinical trials. 


\section{References}

1 Zhou K, Pearson ER. Insights from genome-wide association studies of drug response. Annu. Rev. Pharmacol. Toxicol. 53, 299-310 (2013).

2 Meyer UA, Zanger UM, Schwab M. Omics and drug response. Annu. Rev. Pharmacol. Toxicol. 53, 475-502 (2013).

3 Fu W, O'Connor TD, Jun G et al. Analysis of 6,515 exomes reveals the recent origin of most human proteincoding variants. Nature 493, 216-220 (2013).

4 Roussos P, Mitchell AC, Voloudakis G et al. A role for noncoding variation in schizophrenia. Cell Rep. 9, 1417-29 (2014).

5 Onengut-Gumuscu S, Chen W-G, Burren O et al. Fine mapping of Type 1 diabetes susceptibility loci and evidence for co-localization of causal variants with lymphoid gene enhancers. Nat. Genet. 47, 381386 (2015).

6 Farh KK, Marson A, Zhu J et al. Genetic and epigenetic fine mapping of causal autoimmune disease variants. Nature. 518, 337-343 (2015).

7 Yao L, Tak YG, Berman BP, Farnham PJ. Functional annotation of colon cancer risk SNPs. Nat. Comm. 5, 5114 (2015).

8 Weinhold N, Jacobsen A, Schultz N, Sander C, Lee W. Genome-wide analysis of noncoding regulatory mutations in cancer. Nat. Genet. 46, 1160-1165 (2014).

9 Guenther CA, Tasic B, Luo L, Bedell MA, Kingsley DM. A molecular basis for classic blond hair color in Europeans. Nat. Genet. 46, 748-52 (2014)

10 Cooper GM, Shendure J. Needles in stacks of needles: finding disease-causal variants in a wealth of genomic data. Nat. Rev. Genet. 12, 628-640 (2011).

11 Manolio TA. Genomewide association studies and assessment of the risk of disease. N. Engl. J. Med. 363, 166-176 (2010).

12 Maurano MT, Humbert R, Rynes E et al. Systematic localization of common disease-associated variation in regulatory DNA. Science 337, 1190-1195 (2012).

13 Musunuru K, Strong A, Frank-Kamenetsky M et al. From noncoding variant to phenotype via SORT1 at the 1 p13 cholesterol locus. Nature 466, 714-719 (2010).

14 Parker SCJ, Stitzel ML, Taylor DL et al. Chromatin stretch enhancer states drive cell-specific gene regulation and harbor human disease risk variants. Proc. Natl Acad. Sci. USA 110, 17921-17926 (2013).

15 Mokry M, Middendorp S, Wiegerinck CL et al. Many inflammatory bowel disease risk loci include regions that regulate gene expression in immune cells and the intestinal epithelium. Gastroenterology 146, 1040-1047 (2014).

16 Califano A, Butte AJ, Friend S et al. Leveraging models of cell regulation and GWAS data in integrative networkbased association studies. Nat. Genet. 44, 841-847 (2012).

17 McGeachie MJ, Clemmer GL, Lasky-Su J et al. Joint GWAS Analysis: comparing similar GWAS at different genomic resolutions identifies novel pathway associations with six complex diseases. Genomics Data 2, 202-211

(2014).

18 Sullivan PF1, Daly MJ, O’Donovan M. Genetic architectures of psychiatric disorders: the emerging picture and its implications. Nat. Rev. Genet. 13, 537-551 (2012).

19 Cross-Disorder Group of the Psychiatric Genomics Consortium. Identification of risk loci with shared effects on five major psychiatric disorders: a genome-wide analysis. Lancet 381, 1371-1379 (2013).

20 Kellis M, Wold B, Snyder MP et al. Defining functional DNA elements in the human genome. Proc. Natl Acad. Sci. USA 111, 6131-6138 (2014).

21 Rao SSP, Huntley MH, Durand NC et al. A 3D map of the human genome at kilobase resolution reveals principles of chromatin looping. Cell 159, 1665-1680 (2014).

22 Dixon JR, Selvaraj S, Yue F. Topological domains in mammalian genomes identified by analysis of chromatin interactions. Nature 485, 376-380 (2012).

23 Merkenschlager M, Odom DT. CTCF and cohesin: linking gene regulatory elements with their targets. Cell 152, 1285-1297 (2013).

24 The ENCODE Project Consortium. An integrated encyclopedia of DNA elements in the human genome. Nature 489, 57-74 (2012).

25 Roadmap Epigenomics Consortium. Integrative analysis of 111 reference human epigenomes. Nature 518, 317-330 (2015).

26 Gross DS, Garrard WT. Nuclease hypersensitive sites in chromatin. Annu. Rev. Biochem. 57, 159-197 (1988).

27 Boyle AP, Hong EL, Hariharan M et al. Annotation of functional variation in personal genomes using RegulomeDB. Genome Res. 22, 1790-1797 (2012).

28 Zhou X, Li1 D, Zhang B et al. Epigenomic annotation of genetic variants using the Roadmap Epigenome Browser. Nat. Biotechnol. 33, 345-346 (2015).

29 Selvaraj S, Dixon JR, Bansal V, Ren B. Whole-genome haplotype reconstruction using proximity-ligation and shotgun sequencing. Nat. Biotech. 31, 1111-1118 (2013).

30 Leung D, Jung I, Rajagopal N et al. Integrative analysis of haplotype-resolved epigenomes across human tissues. Nature 518, 350-354 (2015).

31 Hyman SE. Revitalizing psychiatric therapeutics. Neuropsychopharmacol. Rev. 39, 220-229 (2014).

32 Welter D1, MacArthur J, Morales J et al. The NHGRI GWAS Catalog, a curated resource of SNP-trait associations. Nucleic Acids Res. 42, D1001-D1006 (2014).

33 Li MJ, Wang LY, Xia Z, Sham PC, Wang J. GWAS3D: detecting human regulatory variants by integrative analysis of genome-wide associations, chromosome interactions and histone modifications. Nucleic Acids Res. 41, W150-W158 (2013).

34 Li C1, Dong X1, Fan H et al. The 3DGD: a database of genome 3D structure. Bioinformatics 30, 1640-1642 (2014).

35 Lee I, Blom UM, Wang PI, Shim JE, Marcotte EM. Prioritizing candidate disease genes by network-based boosting of genome-wide association data. Genome Res. 21, 1109-1121 (2011). 
Kellis M. Large-scale imputation of epigenomic datasets for systematic annotation of diverse human tissues. Nat. Biotechnol. 33, 364-376 (2015). association study of 14,000 cases of seven common diseases and 3000 shared controls. Nature 447, 661-678 (2007). browser at Washington University. Nat. Methods 8, 989-990 (2011).

Rosenbloom KR, Dreszer TR, Pheasant M et al. ENCODE whole-genome data in the UCSC genome browser. Nucleic Acids Res. 38, D620-D625 (2010).

40 Karolchik D, Barber GP, Casper J et al. The UCSC Genome Browser database: 2014 update. Nucleic Acids Res. 42, D764-D770 (2014).

41 Sunkin SM, Ng L, Lau C et al. Allen Brain Atlas: an integrated spatio-temporal portal for exploring the central nervous system. Nucleic Acids Res. 41, D996-D1008 (2013).

42 Lu Z. PubMed and beyond: a survey of web tools for searching biomedical literature. Database 2011, baq036 (2011).

43 Pomerantz J. Google Scholar and 100 percent availability of information. Info. Tech. Lib. 25, 52-56 (2013). (1992)

45 Park JH, Wacholder S, Gail MH et al. Estimation of effect size distribution from genome-wide association studies and implications for future discoveries. Nat. Genet. 42, 570-575 (2010).

46 Lange LA, Willer CJ, Rich SS. Recent developments in genome and exome-wide analysis of plasma lipids. Curr. Opin. Lipidol. 26, 96-102 (2015).

47 Kato N, Takeuchi F, Tabara Y. Meta-analysis of genome-wide association studies identifies common variants associated with blood pressure variation in east Asians. Nat Genet. 43, 531-538 (2011)

48 Gray KA, Daugherty LC, Gordon SM, Seal RL, Wright MW, Bruford EA. Genenames.org: the HGNC resources in 2013. Nucleic Acids Res. 41, D545-D552 (2012).

49 Sherry ST, Ward MH, Kholodov M et al. dbSNP: the NCBI database of genetic variation. Nucleic Acids Res. 29, 308-311 (2001).

50 Ward LD, Kellis M. HaploReg: a resource for exploring chromatin states, conservation, and regulatory motif alterations within sets of genetically linked variants. Nucleic Acids Res. 40, D930-D934 (2012).

51 Yang TP, Beazley C, Montgomery SB et al. Genevar: a database and Java application for the analysis and visualization of SNP-gene associations in eQTL studies. Bioinformatics 26, 2474-2476 (2010).

52 Lonsdale J, Thomas J, Salvatore M et al. The GenotypeTissue Expression (GTEx) project. Nature Genet. 45, 580-585 (2013).

53 Ernst J, Kellis M. ChromHMM: automating chromatin-state discovery and characterization. Nat. Methods 9, 215-216 (2012).
54 Zhang R, Lahens NF, Ballance HI et al. A circadian gene expression atlas in mammals: implications for biology and medicine. Proc. Natl Acad. Sci. USA 111, 16219-16224 (2014).

55 Willer CJ, Schmidt EM, Sengupta S et al. Discovery and refinement of loci associated with lipid Levels. Nat. Genet. 45, 1274-1283 (2013).

56 Package 'pwr'. https://cran.r-project.org

57 Flicek P, Amode MR, Barrell D et al. Ensembl 2014. Nucleic Acids Res. 42, D749-D755 (2013).

58 ClinicalTrials.gov. www.clinicaltrials.gov

59 Sulem P, Gudbjartsson DF, Geller F et al. Sequence variants at $C Y P 1 A 1-C Y P 1 A 2$ and $A H R$ associate with coffee consumption. Hum. Mol. Genet. 20, 2071-2077 (2011).

60 Amin N, Byrne E, Johnson J et al. Genome-wide association analysis of coffee drinking suggests association with CYP1A1/CYP1A2 and NRCAM. Mol. Psychiatry 17, 1116-1129 (2012).

61 Zanger UM, Schwab M. Cytochrome P450 enzymes in drug metabolism: regulation of gene expression, enzyme activities, and impact of genetic variation. Pharmacol. Ther. 138, 103-141 (2013).

62 Cornelis MC, Monda KL, Yu K et al. Genome-wide meta-analysis identifies regions on 7p21 (AHR) and 15q24 (CYP1A2) as determinants of habitual caffeine consumption. PLoS Genet. 7, e1002033 (2011).

63 Domschke K, Reif A. Behavioral genetics of affective and anxiety disorders. In: Behavioral Neurogenetics. Cryan JF, Reif A (Eds). Springer, Berlin, Heidelberg, Germany 463-502 (2012).

64 Kumasaka N, Aoki M, Okada Y et al. Haplotypes with copy number and single nucleotide polymorphisms in CYP2A6 locus are associated with smoking quantity in a Japanese population. PLoS ONE 7, e44507 (2012).

65 Su T, Bao Z, Zhang QY, Smith TJ, Hong JY, Ding X. Human cytochrome P450 CYP2A13: predominant expression in the respiratory tract and its high efficiency metabolic activation of a tobacco-specific carcinogen, 4-(methylnitrosamino)-1-(3-pyridyl)-1-butanone. Cancer Res. 60, 5074-5079 (2000).

66 Tobacco and Genetics Consortium. Genome-wide metaanalyses identify multiple loci associated with smoking behavior. Nat Genet. 42, 441-447 (2010).

67 Hoft NR, Stitzel JA, Hutchison KE, Ehringe MA. CHRNB2 promoter region: association with subjective effects to nicotine and gene expression differences. Genes Brain Behav. 10, 176-185 (2011).

68 Timofeeva MN, McKay JD, Smith GD et al. Genetic polymorphisms in 15q25 and 19q13 loci, cotinine levels, and risk of lung cancer in EPIC. Cancer Epidemiol. Biomarkers Prev. 20, 2250-2261 (2011).

69 Enoch MA, Rosser AA, Zhou Z, Mash DC, Yuan Q, Goldman D. Expression of glutamatergic genes in healthy humans across 16 brain regions; altered expression in the 
hippocampus after chronic exposure to alcohol or cocaine. Genes Brain Behav. 13, 758-776 (2014).

70 Nelson EC, Lynskey MT, Heath AC et al. Association of OPRD1 polymorphisms with heroin dependence in a large case-control series. Addiction Biol. 19, 111-121 (2014).

71 Pandit S, Zhou Y, Shiue L et al. Genome-wide analysis reveals SR protein cooperation and competition in regulated splicing. Mol. Cell 50, 223-235 (2013).

72 Kertes DA, Kalsi G, Prescott CA et al. Neurotransmitter and neuromodulator genes associated with a history of depressive symptoms in individuals with alcohol dependence. Alcohol. Clin. Exp. Res. 35, 496-505 (2011).

73 Blum K, Fornari F, Downs BW et al. Genetic Addiction Risk Score (GARS): Testing for Polygenetic Predisposition and Risk to Reward Deficiency Syndrome (RDS). InTech Open Access Publisher (2011).

74 Liu Z, Guo X, Jiang Y, Zhang H. NCK2 is significantly associated with opiates addiction in African-origin men. Scientific World Journal. 2013, 748979 (2013).

75 Huroy S. Mechanisms of EphB2 mediated opiate-dependent tolerance and learning [MS thesis]. University of Toronto, ON, Canada (2012)

76 Baik I, Cho NH, Kim SH, Han BG, Shin C. Genome-wide association studies identify genetic loci related to alcohol consumption in Korean men. Am. J. Clin. Nutr. 93, 809-816 (2011).

77 Cederbaum AI. Alcohol metabolism. Clin. Liver Dis. 16, 667-685 (2012).

78 Duell EJ, Sala N, Travier N et al. Genetic variation in alcohol dehydrogenase (ADH1A, ADH1B, ADH1C, ADH7) and aldehyde dehydrogenase (ALDH2), alcohol consumption and gastric cancer risk in the European Prospective Investigation into Cancer and Nutrition (EPIC) cohort. Carcinogenesis 33, 361-367 (2012).

79 Lind PA, Zhu G, Montgomery GW et al. Genome-wide association study of a quantitative disordered gambling trait. Addiction Biol. 18, 511-522 (2013).

80 Razvodovsky Y, Borodinsky A, Pascual-Mora M et al. Basic research. Alcohol and Alcoholism, 48 (Suppl. 1), i41-i46 (2013).

81 Jagannathan L, Swaminathan K, Kumar SM, Kumar GR, Dey A. Bio-informatics based analysis of genes implicated in alcohol mediated liver injury. Gene 494, 130-139 (2012).

82 Wang $\mathrm{C}, \mathrm{Xu}$ Y, Feng X et al. Linkage analysis and wholeexome sequencing exclude extra mutations responsible for the parkinsonian phenotype of spinocerebellar ataxia-2. Neurobiol. Aging 36, 545.e1-e7 (2015).

83 Modig F, Patel M, Magnusson M, Fransson PA. Study II: mechanoreceptive sensation is of increased importance for human postural control under alcohol intoxication. Gait Posture 35, 419-427 (2012).

84 Zuko A, Kleijer KT, Oguro-Ando A et al. Contactins in the neurobiology of autism. Eur. J. Pharmacol. 719, 63-74 (2013).

85 Verpelli C, Galimberti I, Gomez-Mancilla B, Sala C. Molecular basis for prospective pharmacological treatment strategies in intellectual disability syndromes. Dev. Neurobiol. 74, 197-206 (2014).
86 Mitchell KJ. The genetics of neurodevelopmental disease. Curr. Opin. Neurobiol. 21, 197-203 (2012).

87 Berezin V, Walmod PS. Cell Adhesion Molecules: Implications in Neurological Diseases. Vol. 8. Springer Science \& Business Media, NY, USA (2013).

88 Mehta D, Klengel T, Conneely KN et al. Childhood maltreatment is associated with distinct genomic and epigenetic profiles in posttraumatic stress disorder. Proc. Natl Acad. Sci. USA 110, 8302-8307 (2013)

89 Mullins N, Perroud N, Uher R et al. Genetic relationships between suicide attempts, suicidal ideation and major psychiatric disorders: a genome-wide association and polygenic scoring study. Am. J. Med. Genet. 165(5), 428-437 (2014).

90 Gelernter J, Kranzler HR, Sherva R et al. Genome-wide association study of nicotine dependence in american populations: identification of novel risk loci in both AfricanAmericans and European-Americans. Biol. Psychiatry 77(5), 493-503 (2014).

91 Dallaspezia S, Poletti S, Lorenzi C, Pirovano A, Colombo C, Benedetti F. Influence of an interaction between lithium salts and a functional polymorphism in SLC1A2 on the history of illness in bipolar disorder. Mol. Diagn. Ther. 16, 303-309 (2012).

92 Djurovic S, Gustafssonc O, Mattingsdala M et al. A genomewide association study of bipolar disorder in Norwegian individuals, followed by replication in Icelandic sample. J. Affect. Disord. 126, 312-316 (2010).

93 Thier S, Lorenz D, Nothnagel M et al. Polymorphisms in the glial glutamate transporter SLC1A2 are associated with essential tremor. Neurology 79, 243-248 (2012).

94 Aberg K, Adkins DE, Liu Y et al. Genome-wide association study of antipsychotic-induced QTc interval prolongation. Pharmacogenomics 12, 165-72 (2012).

95 Ostrousky O, Meged S, Loewenthal R et al. NQO2 gene is associated with clozapine-induced agranulocytosis. Tissue Antigens 62, 483-491 (2003).

96 Miettinen TP, Björklund M. NQO2 is a reactive oxygen species generating off-target for acetaminophen. Mol. Pharm. 11, 4395-4404 (2014).

97 Chang KC, Sasano T, Wang YC, Huang SKS. Nitric oxide synthase 1 adaptor protein, an emerging new genetic marker for QT prolongation and sudden cardiac death. Acta Cardiol. Sin. 29, 217-225 (2013).

98 Jamshidi Y, Nolte IM, Dalageorgou C et al. Common variation in the NOS1AP gene is associated with druginduced QT prolongation and ventricular arrhythmia. J. Am. Coll. Cardiol. 60, 841-850 (2012).

99 Kapoor A, Sekar RB, Hansen NF et al. QT IntervalInternational GWAS Consortium: an enhancer polymorphism at the cardiomyocyte intercalated disc protein NOS1AP locus is a major regulator of the QT interval. Am. J. Hum. Genet. 94, 854-869 (2014).

100 Tomás M, Napolitano C, De Giuli L et al. Priori Polymorphisms in the NOS1AP gene modulate QT interval duration and risk of arrhythmias in the long QT syndrome. J. Am. Coll. Cardiol. 55, 2745-2752 (2010). 
101 Adkins DE, Åberg K, McClay JL et al. Genomewide pharmacogenomic study of metabolic side effects to antipsychotic drugs. Mol. Psychiatry 16, 321-332 (2011).

102 Kolder IC. Family-based genome-wide association analysis for the identification of genetic modifiers of heart rate and electrocardiographic indices of conduction and repolarization in a large Dutch family with a mutation in SCN5A. Genetic modifiers in familial cardiac rhythm disorders, 105 (2012).

103 Libbrecht MW, Ay F, Hoffman MM, Gilbert DM, Bilmes JA, Noble WS. Joint annotation of chromatin state and chromatin conformation reveals relationships among domain types and identifies domains of cell-type-specific expression. Genome Res. 25, 1-14 (2015).

104 Hoffman MM, Buske OJ, Wang J, Weng Z, Bilmes JA, Noble WS. Unsupervised pattern discovery in human chromatin structure through genomic segmentation. Nat. Methods 9, 473-476 (2012).

105 Zanger UM, Klein K, Thomas M et al. Genetics, epigenetics and regulation of drug metabolizing cytochrome $\mathrm{P} 450$ enzymes. Clin. Pharmacol. Ther. 95, 258-261 (2014).

106 Schaub MA, Boyle AP, Kundaje A, Batzoglou S, Snyder M. Linking disease associations with regulatory information in the human genome. Genome Res. 22, 1748-1759 (2012).

107 Hawrylycz MJ, Lein ES, Guillozet-Bongaarts AL et al. An anatomically comprehensive atlas of the adult human brain transcriptome. Nature 489, 391-399 (2012).

108 Kang HJ, Kawasawa YI, Cheng F et al. Spatio-temporal transcriptome of the human brain. Nature 478, 483-489 (2011).

109 Aguilar-Arnal L, Sassone-Corsi P. Chromatin landscape and circadian dynamics: Spatial and temporal organization of clock transcription. Proc. Natl Acad. Sci. USA 112, 6863-6870 (2014).

110 Borel C, Ferreira PG, Santoni F et al. Biased allelic expression in human primary fibroblast single cells. Am. J. Hum. Genet. 96, 1-11 (2015).

111 Deng Q, Ramskold D, Reinius B et al. Single-cell RNA-seq reveals dynamic, random mono-allelic gene expression in mammalian cells. Science 343, 193-196 (2014).

112 Osborne CS, Chakalova L, Brown KE et al. Active genes dynamically co-localize to shared sites of ongoing transcription. Nat. Genet. 36, 1065-1071 (2004).
113 Palacios R, Gazave E, Goñi J et al. Allele-specific gene expression is widespread across the genome and biological processes. PLoS ONE 4, e4150 (2009).

114 Schalkwyk LC, Meaburn EL, Smith R et al. Allelic skewing of DNA methylation is widespread across the genome. Am. J. Hum. Genet. 86, 196-212 (2010).

115 McDaniell R, Lee BK, Song L et al. Heritable individualspecific and allele-specific chromatin signatures in humans. Science 328, 235-239 (2010).

116 Park J, Gail MH, Weinberg CR et al. Distribution of allele frequencies and effect sizes and their interrelationships for common genetic susceptibility variants. Proc. Natl Acad. Sci. USA 108, 18026-18031 (2011).

117 Stringer S, Wray NR, Kahn RS, Derks EM. Underestimated effect sizes in GWAS: fundamental limitations of single SNP analysis for dichotomous phenotypes. PLoS ONE 6, e27964 (2011).

118 Brownstein MJ. A brief history of opiates, opioid peptides, and opioid receptors. Proc. Natl Acad. Sci. USA 90, 5391-5393 (1993).

119 Crocq M. Historical and cultural aspects of man's relationship with addictive drugs. Dialogues Clin Neurosci. 9, 355-361 (2007).

120 Edwards SL, Beesley J, French JD, Dunning M. Beyond GWAS: illuminating the dark road from association to function. Am. J. Hum. Genet. 93, 779-797 (2013).

121 Benjamini Y, Hochberg B. Controlling the false discovery rate: a practical and powerful approach to multiple testing. J. Royal Stat. Soc. 57(1), 289-300 (1995).

122 Lee SH, Yang J, Goddard ME, Visscher PM, Wray NR. Estimation of pleiotropy between complex diseases using single-nucleotide polymorphism-derived genomic relationships and restricted maximum likelihood. Bioinformatics 28, 2540-2542 (2012).

123 Jia P, Wang L, Meltzer HY, Zhaoa Z. Common variants conferring risk of schizophrenia: a pathway analysis of GWAS data. Schizophr. Res. 122, 38-42 (2010).

124 Shi G, Boerwinkle E, Morrison AC, Gu CC, Chakravarti A, Rao DC. Mining gold dust under the genome wide significance level: a two-stage approach to analysis of GWAS. Genet. Epidemiol. 35, 111-118 (2011). 